\title{
Review on Relationship between Psychological Contract and Organizational Citizenship Behavior
}

\author{
Li Chu ${ }^{1, a^{*}}$, Bixiang Zhu ${ }^{2, b}$ \\ $1^{2}$ School of Economics and Management, Nanjing University of Science and Technology, \\ Nanjing 210094, China \\ achuli905@163.com, bzhubixiang@tom.com
}

\begin{abstract}
With the increasing competition among enterprises, the study of psychological contract and organizational citizenship behavior between enterprises and employees has always been the concern of management scholars. On the basis of a brief review of the research on psychological contract and organizational citizenship behavior, focusing on the relationship between psychological contract and organizational citizenship behavior, this paper ruptured and summarized the relevant research on the effect of psychological contract on organizational citizenship behavior, the effect of psychological contract violation on organizational citizenship behavior and the impact of organizational citizenship behavior on psychological contract, so as to have a certain reference on further study of the relationship between psychological contract and organizational citizenship behavior in the new era background.
\end{abstract}

Keywords: Psychological contract; Organizational citizenship behavior; Relationship

\section{心理契约与组织公民行为关系研究综述}

\author{
储 丽 $^{1}$, 朱必祥 ${ }^{2}$ \\ （南京理工大学 经济管理学院、企业管理系，江苏南京 210094）
}

摘要: 随着企业间竞争的日益加剧，对企业与员工之间的心理契约和组织公民行为的研究一直以来都颇受管理学界的关 注。本文在对心理契约、组织公民行为的相关研究进行简单回顾的基础上, 着眼于心理契约与组织公民行为之间的关系, 从 心理契约履行对组织公民行为的影响、心理契约违背及破裂对组织公民行为的影响和组织公民行为对心理契约的影响三个方 面对相关的研究加以梳理, 并从整体上进行了总结, 以期对新时代背景下心理契约与组织公民行为关系的深入研究有一定的 借鉴意义。

关键词: 心理契约; 组织公民行为; 关系

中图分类号：F241 文献标识码：A

引言

20 世纪 90 年代以来, 全球竞争的加剧使组织所处的环境日趋不确定和复杂, 雇佣关系也随之发生了 深刻的变化。在竞争性和变化性日益增强的环境中，组织要应对双重挑战一既要增强对环境的适应性，又 要通过激励员工充分履行角色内行为和角色外行为来提升组织绩效, 就必须思考如何建立良性的雇佣关

系，由此也吸引了众多国内外学者对心理契约、组织公民行为及其之间关系的多角度研究。

本文在全面梳理国内外心理契约与组织公民行为关系的基础上，综述已经取得的成果，分析以往存在 的问题，并对未来研究方向作出展望，以期为该领域日后更为深入的研究提供借鉴和帮助。

\section{1 心理契约研究概述}

\section{1 心理契约的概念界定}

Argyris 在《理解组织行为》一书中首次提出心理的工作契约这一概念 ${ }^{[1]}$ 。Levinson 等通过实证研究， 
证实了雇主与雇员之间确实存在着心理契约, 并将这一概念定义为关系双方可能并未清楚意识到的,但却 是统摄双方关系的一系列的相互期望 ${ }^{[2]}$ 。到 20 世纪 80 年代后期,针对如何确定组织水平上的心理契约的争 论, 出现了关于心理契约广义与狭义两个层面的概念界定。(1) 以 Guest, Herriot, Pemberton 等人为代表, 强调遵循 Schein 等人提出心理契约概念的原意, 认为心理契约是雇佣双方对交换关系中彼此责任和义务的 主观理解, 即对心理契约的广义概念界定, 被称为心理契约理论的古典学派 ${ }^{[3]}{ }^{[4]}$ 。(2) 以 Rousseau, Robinson, Morrison 等人为代表, 强调心理契约是雇员个体对双方交换关系中彼此义务的主观理解, 即对心理契约的狭 义概念界定, 被称为心理契约理论的卢梭学派 ${ }^{[5][6]}$ 。

\section{2 心理契约的结构维度}

对心理契约的维度划分, 国内外目前还没有形成统一的结论。Millward 等通过量表调查分析,结果证实 了交易契约与关系契约的存在 ${ }^{[7]}$ 。Kickul 等通过因素分析的实证研究方法,提取了雇主责任的外在契约和内 在契约 2 个因素 ${ }^{[8]}$ 。陈加洲等对中国企业员工与组织之间的心理契约进行了理论研究和实证研究,也发现了 两个因素, 他们将之命名为现实责任和发展责任 ${ }^{[9]}$ 。Lee 等对香港和美国工作团队的研究认为员工心理契 约由关系因素、交易因素和团队成员因素 3 个维度构成 ${ }^{[10]}$ 。李原研究发现中国企业员工的心理契约由 3 个 维度构成:规范性责任、人际型责任和发展型责任 ${ }^{[1]}$ 。

\section{2 组织公民行为研究概述}

\section{1 组织公民行为的概念界定}

西方学者对组织公民行为的研究可以追溯到 Bamard 在 1938 年提出的想要合作的意愿。1966 年, Katz 和 Kahn 提出组织公民的概念。他们认为一个运作良好的组织需要存在三种行为方式: (1) 引导并把员工 留在组织中; (2) 完成工作要求的职责; (3) 角色要求之外的创造性、自发行为。第三种行为方式就是组 织公民行为的倠形。1 1983 年, Bateman 和 Organ 将第三种行为称为“组织公民行为”。1988 年, Organ 把组 织公民行为正式定义为“员工的自主行为, 没有直接或详细的在正式报酬体系中规定, 能在整体上提高组 织绩效 ${ }^{[12]}$ 。

\section{2 组织公民行为的结构维度}

自从 Organ 正式提出组织公民行为的概念以来, 国内外学者对于其特征维度的构成提出了各自的观点, 但各派众说纷纭仍未达成最后的一致。1983 年 Smith 等人依据一个 16 项调查表的调查结果抽取出了 OCB 二维结构一利他和一般顺从。至今, 已被定义过的 OCB 特征维度多达三十多项, 主要有二维结构、四维 结构、五维结构和七维结构。国内最早对 OCB 特征维度研究的是台湾学者林淑姬。1992 年, 她提出 OCB 的特征维度有六个: 认同组织、协助同事、不生事争利、公私分明、敬业守法、自我充实 ${ }^{[13]}$ 。2000 年, 樊 景立采用同样的研究方法探索了中国大陆的组织公民行为, 提出了四层面十维度结构: 积极主动、帮助同 事、意见表露、参与集体活动、维护组织形象、自觉学习、参与公益活动、维护和节约组织资源、保持工 作环境整洁、人际和谐 ${ }^{[14]}[15]$ 。

\section{3 心理契约与组织公民行为关系研究综述}

\section{1 心理契约履行对组织公民行为的影响}

罗秋明的研究指出, 心理契约是影响组织公民行为的一个重要变量, 心理契约与组织公民行为之间存 在正相关, 心理契约的履行程度对组织公民行为有显著影响, 心理契约通过组织公平和组织支持感从而影 
响组织公民行为 ${ }^{[16]}$ 。

林澜通过对 345 名高校教师的问卷调查, 发现教师心理契约中的学校已履行责任和学校未来责任对其 学校公益行为、同事公益行为、学生公益行为的履行有显著正向的预测作用, 结果验证了学校已履行责任 激发教师反应式回报，教师对学校未来责任履行的预期激发其前瞻式回报，前瞻式回报的动机强于反应 式回报，从而探讨出了心理契约对组织公民行为的影响机制 ${ }^{[17]}$ 。

于桂兰等人以 57 项实证研究为对象，综合使用元分析、路径分析及回归分析等方法，对心理契约与 组织公民行为的关系进行量化探讨, 并选取了心理契约履行与违背两个方向。结果表明:（1）心理契约履 行与组织公民行为正相关，心理契约违背与组织公民行为负相关。（2）二者的主要中介变量中，信任起主 要作用 ${ }^{[18]}$ 。

程志伟通过实证研究的范式, 以酒店销售人员为研究对象, 得出以下结论:（1）总体上心理契约对组 织公民行为产生正向影响;（2）心理契约对利他行为、个人主动性和公司认同产生正向影响;（3）心理契 约对内在利益获得产生负向影响; (4) 心理契约对销售人员的组织公民行为影响有限 ${ }^{[19]}$ 。

\section{2 心理契约违背及破裂对组织公民行为的影响}

赵否等人采用问卷调查法, 研究了同事支持感在心理契约破裂和组织公民关系间的调节作用。研究结 果表明: 同事支持感会削弱心理契约破裂和组织公民行为的关系的强度, 即当个体感知到较高程度的同事 支持时，虽然个体感受到心理契约被破坏，也会维持较高程度的组织公民行为 ${ }^{[20]}$ 。

陈学军等人通过对 16 家企业 225 名员工的问卷调查, 实证检验了心理契约违背、上级支持感和组织 公民行为之间的关系, 结果发现:（1）心理契约违背对上级支持感存在显著负面影响;（2）上级支持感在 关系型契约违背与员工组织公民行为的关系中起着中介作用;（3）交易型契约违背对组织公民行为不存在 影响 ${ }^{[21]}$ 。

王丽平和于志川根据员工与企业的社会交换动态过程, 引入后悔这一中介变量, 解释心理契约破裂对 组织公民行为的影响机理。通过以知识型员工为样本的实证研究, 证实了后悔在心理契约破裂影响除组织 认同的其他组织公民行为四个维度中均发挥了部分中介作用。根据实证结果, 解释了特殊情境下的心理契 约破裂对组织公民行为负向影响不显著的原因, 扩充了现有对心理契约破裂与组织公民行为的研究, 为管 理者改进组织公民行为水平提供借鉴 ${ }^{[22]}$ 。

台湾学者 Chu-Mei Liu 等人以台湾的酒店业为研究对象, 得出如下结论: 心理契约破裂对组织信任感 和组织公民行为有着显著的负向影响。组织信任感对组织公民行为有着显著的正向影响, 组织信任感是一 个中介变量 ${ }^{[23]}$ 。

\section{3 组织公民行为对心理契约的影响}

罗秋明的研究指出, 组织公民行为是心理契约履行状态的晴雨表。（1）组织公民行为可以反映员工与 组织心理契约的变化状态。通过观察员工的组织公民行为, 特别是组织公民行为的变化, 更能够清楚地反 映出员工与组织的社会交换关系的变化, 更能够清楚地了解心理契约管理中的问题。(2) 组织公民行为可 以强化员工的心理契约。组织中, 员工如果表现出主动承担角色外的行为, 主动为组织奉献, 这种行为又会 随着时间的推移逐渐积累而最终能够提升组织绩效, 那么管理者会认同这种行为并给与员工高评价, 进而 可能是员工获得奖励、加薪、晋升等方面的重要依据, 这种结果又会反过来巩固和强化员工的心理契约 ${ }^{[16]}$ 。 


\section{4 结语}

整合现有的研究，我们可以总结出心理契约与组织公民行为之间是相互联系，相互影响的关系。心理 契约是组织公民行为的重要变量, 心理契约履行与组织公民行为呈正相关, 心理契约履行程度不同, 企业 内的组织公民行为则存在着显著的差异，心理契约的违背及破裂则对组织公民行为产生较为显著的负向影 响。而反过来, 组织公民行为则是企业心理契约履行程度的晴雨表, 对心理契约有着强化作用。纵向来看, 自 20 世纪 90 年代以来, 国内外对于心理契约与组织公民行为关系的研究范围日益广泛, 研究主体日益多 元, 研究视角日益多样, 研究程度日益深入, 研究成果也日益丰富。横向来看, 国外的研究起步较早, 取 得的研究成果也更为丰富和多样, 而中国引入心理契约及组织公民行为概念的时间并不长, 大多数的研究 仍然建立在国外研究的基础之上, 国内外仍然存在着差距。

虽然对心理契约与组织公民行为关系的研究已开展了二十多年, 我们对两者关系的认识越来越深入, 但研究中存在的问题也日益凸显。根据对以往的研究成果和存在问题的梳理分析, 我们认为未来的研究趋 势有以下两点:

第一, 研究范围将进一步拓展, 呈现出多层面立体交叉的研究格局。以往对心理契约及组织公民行为 的研究主要集中在营利组织, 实际上非营利组织同样也存在着心理契约及组织公民行为的问题, 而且在各 类组织中个体、群体和组织不同层面两两之间均存在着心理契约和组织公民行为之间的关系。因此我们预 计, 对各类组织各类主体之间的心理契约与组织公民行为之间关系的广泛研究将是未来的一个发展趋势。

第二, 在理论研究与实证研究互动的基础上, 进一步理清两者关系的理论框架。对于心理契约与组织 公民行为关系形成机理的研究由于缺乏研究手段, 目前涉足的学者很少, 仍然处于模糊混沌的阶段, 未来需 要开展大量的实证研究来检验现有的一些理论假设, 在理论研究与实证研究的互动中逐步构建起较为完善 的理论体系框架。

\section{参考文献:}

[1] Rodrigues S B, Child J. Private equity, the minimalist organization and the quality of employment relations[J]. Human Relations, 2010, 63(9):1321-1342.

[2] Li J, Dai L. A Review of Psychological Contract[J]. Psychology, 2015: 1539-1544.

[3] Cardona Echeverri D R, Zambrano Cruz R. Review of organizational climate assessment tools [M]. Springer Netherlands, 2011.

[4] Cardona Echeverri D R, Zambrano Cruz R. Review of organizational climate assessment tools [M]. Springer Netherlands, 2011.

[5] Demirkasimoglu N. Teachers' Psychological Contract Perceptions and Person-Environment Fit Levels[J]. Eurasian Journal of Educational Research, 2014: 23.

[6] Brande V D, Janssens M, Sels L, et al. Multiple types of psychological contracts: a six-cluster solution[J]. Human Relations, 2003, 56(11): 1349-1378.

[7] Landells E M, Albrecht S L. The Positives and Negatives of Organizational Politics: A Qualitative Study [J]. Journal of Business \& Psychology, 2015: 1-18.

[8] Chen S Y, Jin F U, Chen C C. Implementing high performance HR practices in Asia: HR practice consistency, employee roles, and performance[J]. Asia Pacific Journal of Management, 2016: 1-22.

[9] 陈加洲, 凌文辁, 方俐洛. 员工心理契约结构维度的探索与验证 $[J]$. 科学学与科学技术管理, 2004 (3)：94-97. 
[10] Hui C, Kwong J Y Y, Lee C. Work Motivation in Asia[J]. Handbook of Asian Management, 2004: 349-372.

[11] 李原. 员工心理契约的结构及相关因素研究 [D]. 北京：首都师范大学教育科学学院, 2002.

[12] Finkelstein M A. Correlates of Individualism and Collectivism: Predicting Organizational Citizenship Behavior[J]. International Journal of Psychology \& Behavioral Sciences, 2013, 3(2) : 57-62.

[13］林淑姬. 薪酬公平、程序公平与组织承诺、组织公民行为关系之研究 [D]. 台湾国立政治大学企业管理研究所博士 论文, 1992: 34-55.

[14] Teh P L, Yong C C. Knowledge Sharing in is Personnel: Organizational Behavior's Perspective[J]. Journal of Computer Information Systems, 2011, 51(4): 11-21.

[15] Farh J L, Zhong C B, \&Organ D W. Organizational Citizenship Behavior in the People' s Republic of China[J]. Organization Science, 2004, 15(2): 241-253.

[16] 罗秋明. 论心理契约与组织公民行为的关系 [J]. 湖南工业大学学报, 2009, 14(4)：4-7.

[17］林澜. 反应式回报与前瞻式回报：心理契约对组织公民行为的影响机制 [J]. 经济管理, 2012，34 (6)：65-74.

[18] 于桂兰，陈明，于楠. 心理契约与组织公民行为的关系一元分析回顾及样本选择与测量方法的调节作用 [J]. 吉 林大学社会科学学报, 2013, 53(2)：115-123.

[19］程志伟. 心理契约与组织公民行为的影响研究 [J]. 中国管理现代化，2014，17(11)：67-68.

[20] 赵否, 沈伊默, 魏春梅, 等. 心理契约破坏对组织公民行为的影响：同事支持感的调节作用 $[\mathrm{J}]$. 心理学探新, 2011, $31(6)$ : 549-553.

[21] 陈学军, 章倩, 陈刚. 心理契约违背对组织公民行为的影响：上级支持的中介作用 $[J]$. 人类工效学, 2011, $17(2): 19-23$.

[22］王丽平，于志川。心理契约破坏对组织公民行为影响研究：基于后悔的中介作用 [J]. 预测，2012，31(3)： 24-29.

[23] Chu-Mei Liu, Chien-Jung Huang, Kai-Ping Huang, et al. Psychological contract breach, organizational trust and organizational citizenship behavior of hotel industry in Taiwan[J]. Pakistan Journal of Statistics, 2013, $29(5):$ 635-648.

\section{References}

[1] Rodrigues S B, Child J. Private equity, the minimalist organization and the quality of employment relations [J]. Human Relations, 2010, 63(9):1321-1342.

[2] Li J, Dai L. A Review of Psychological Contract [J]. Psychology, 2015: 1539-1544.

[3] Cardona Echeverri D R, Zambrano Cruz R. Review of organizational climate assessment tools [M]. Springer Netherlands, 2011.

[4] Cardona Echeverri D R, Zambrano Cruz R. Review of organizational climate assessment tools [M]. Springer Netherlands, 2011.

[5] Demirkasimoglu N. Teachers' Psychological Contract Perceptions and Person-Environment Fit Levels [J]. Eurasian Journal of Educational Research, 2014: 23.

[6] Brande V D, Janssens M, Sels L, et al. Multiple types of psychological contracts: a six-cluster solution [J]. Human Relations, 2003, 56(11): 1349-1378.

[7] Landells E M, Albrecht S L. The Positives and Negatives of Organizational Politics: A Qualitative Study [J]. Journal of Business \& Psychology, 2015: 1-18.

[8] Chen S Y, Jin F U, Chen C C. Implementing high performance HR practices in Asia: HR practice consistency, employee roles, and performance [J]. Asia Pacific Journal of Management, 2016: 1-22. 
[9] Chen Jiazhou Lingwenquan Fang Liluo Exploration and Verification of the Dimension of Employee's Psychological Contract Structure [J]. Science in Science and Technology Management, 2004 (3): 94-97.

[10]Hui C, Kwong J Y Y, Lee C. Work Motivation in Asia [J]. Handbook of Asian Management, 2004: 349-372.

[11]Li Yuan Research on the Structure and Related Factors of Psychological Contract of Employees [D]. Beijing: Capital Normal University, School of Educational Science, 2002.

[12]Finkelstein M A. Correlates of Individualism and Collectivism: Predicting Organizational Citizenship Behavior [J]. International Journal of Psychology \& Behavioral Sciences, 2013, 3(2): 57-62.

[13]Lin Shuji Compensation Equity, Fairness of Program and Organizational Commitment, Organizational Citizenship Behavior [D]. Doctoral Dissertation, Institute of Enterprise Management, National University of Taiwan, 1992: 34-55.

[14] Teh P L, Yong C C. Knowledge Sharing in is Personnel: Organizational Behavior's Perspective [J]. Journal of Computer Information Systems, 2011, 51(4): 11-21.

[15]Farh J L, Zhong C B, \&Organ D W. Organizational Citizenship Behavior in the People's Republic of China [J]. Organization Science, 2004, 15(2): 241-253.

[16]Luo Qiuming Discussion on the Relationship between Psychological Contract and Organizational Citizenship [J]. Journal of Hunan University of Technology, 2009, 14 (4): 4-7.

[17]Lin Lan Responsive Return and Prospective Return: The Mechanism of Psychological Contract on Organizational Citizenship [J]. Economic Management, 2012, 34 (6): 65-74.

[18] Yu Guilan Chen Ming Yunan Relationship between Psychological Contract and Organizational Citizenship Behavior: Review of Meta-analysis and Regulation of Sample Selection and Measurement Method [J]. Journal of Jilin University Social Science, 2013, 53 (2): 115-123.

[19]Cheng Zhiwei A Study on the Effects of Psychological Contract and Organizational Citizenship Behavior [J]. China Management Modernization, 2014, 17 (11): 67-68.

[20]Zhao Lei Shen Yimo Wei Chunmei,etc. The Impact of Psychological Contract Destruction on Organizational Citizenship: The Role of Counselor's Support [J]. Psychology Exploration, 2011, 31 (6): 549-553.

[21]Chen Xuejun Zhang Qian Chen Gang The Impact of Psychological Contract Violation on Organizational Citizenship: Mediating Role of Higher Support [J]. Ergonomics, 2011, 17 (2): 19-23.

[22]Wang Liping Yu Zhichuan A Study on the Impact of Psychological Contract Damage on Organizational Citizenship Behavior: Based on the Mediating Role of Regret [J]. Forecast, 2012, 31 (3): 24-29.

[23]Chu-Mei Liu, Chien-Jung Huang, Kai-Ping Huang, et al. Psychological contract breach, organizational trust and organizational citizenship behavior of hotel industry in Taiwan[J]. Pakistan Journal of Statistics, 2013, 29(5): 635-648. 